Sumat Pramer

\title{
Review on cases in hepatobiliary and pancreatic imaging:
}

This is very comprehensive, well illustrated book is written by the radiologist from ILBS, New Delhi.

Tremendous advances have been made in recent years in imaging of and interventional techniques for biliary and pancreatic disease. Hepatobiliary radiology has become a subspecialty of great interest to radiologists.

This book is well organized into easy-to-read case based review with excellent detail and provides high-quality illustrations, figures and references

Cases in Hepatobiliary and Pancreatic Imaging is very practical, comprehensive case based approach, an excellent reference for any radiologist who has an interest in hepatobiliary and pancreatic disease.

This book is recommended for junior radiologists, gastroenterologists and those want to become more proficient in hepatobiliary and pancreatic imaging.

\section{Dr Shivanand Gamanagatti}

Additional Professor of Radiology

All India Institute of Medical Sciences

New Delhi-110029

Email: shiv223@ rediffmail.com 